\title{
IRREDUCIBLE SUBGROUPS OF SYMPLECTIC GROUPS IN CHARACTERISTIC 2
}

\author{
CHRISTOPHER PARKER and PETER ROWLEY
}

(Received 13 January 2000; revised 25 July 2001)

Communicated by R. B. Howlett

\begin{abstract}
Suppose that $V$ is a finite dimensional vector space over a finite field of characteristic 2, $G$ is the symplectic group on $V$ and $a$ is a non-zero vector of $V$. Here we classify irreducible subgroups of $G$ containing a certain subgroup of $\mathrm{O}_{2}\left(\mathrm{Stab}_{G}\langle a\rangle\right)$ all of whose non-trivial elements are 2-transvections.
\end{abstract}

2000 Mathematics subject classification: primary 20CDE.

Keywords and phrases: symplectic group, orthogonal group, 2-transvections, transvections.

\section{Introduction}

Let $k$ denote the Galois field GF( $q)$, where $q=2^{m}$, and suppose $V$ is a finite dimensional vector space over $k$. An involution $g$ of $\mathrm{GL}(V)$ is a transvection (respectively a 2-transvection) of $V$ if $C_{V}(g)$ has codimension 1 (respectively 2) in $V$. A subgroup $K$ of $\mathrm{GL}(V)$ is called a transvection subgroup if $C_{V}(K)$ has codimension $1,[V, K]$ has dimension 1 , and $K$ is isomorphic to the additive group of $k$. Assume, additionally, that $\operatorname{dim} V=2 n$ where $n \geq 2$ and $f$ is a non-degenerate alternating bilinear form on $V$. Letting $G$ denote $\operatorname{Sp}(V)$, the symplectic group on $V$ defined by $f$, we may now state our main result.

THEOREM 1.1. Let a be a non-zero vector of $V$ and put $H=\mathrm{Stab}_{G}\langle a\rangle$. Suppose that $X$ is a subgroup of $O_{2}(H)$ which satisfies

(i) $|X|=q^{2 n-2}$; and

(ii) no element of $X$ acts as a transvection on $V$.

(C) 2002 Australian Mathematical Society $1446-7887 / 2000 \$ A 2.00+0.00$ 
If $L$ is a subgroup of $G$ which contains $X$ and acts irreducibly on $V$, then $L$ acts naturally on $V$ as one of $\mathrm{Sp}(V), \Omega^{ \pm}(V), \mathrm{O}^{ \pm}(V)$ or $q=n=2$ and $L \cong \operatorname{Alt}(6) \cong$ $\mathrm{Sp}_{4}(2)^{\prime}$ or $\Gamma \mathrm{L}(2,4) \cong \mathrm{Sym}(5)$.

We remark that the listed groups all satisfy the hypothesis of Theorem 1.1. The exceptional cases when $q=n=2$ are caused by the non-simplicity of $\operatorname{Sp}_{4}(2) \cong$ $\operatorname{Sym}(6)$ in the Alt(6) case and by the fact that Sym(6) has two classes of subgroups isomorphic to Sym(5) in the second case. To clarify the second case further take $V$ to be the doubly deleted GF(2)-permutation module for $\operatorname{Sym}(6)$, the point stabilizer $\operatorname{Sym}(5)$ acts on $V$ as $\mathrm{O}_{4}^{-}(2)$, while the transitive $\operatorname{Sym}(5)$ when restricted to $\operatorname{Alt}(5)$ acts on $V$ as $\mathrm{SL}_{2}(4)$.

Observe that, as the non-trivial elements of the elementary abelian 2-group $O_{2}(H)$ are either transvections or 2-transvections, every non-trivial element of $X$ must act as a 2-transvection on $V$. So Theorem 1.1 may be seen as a kindred spirit to the results of McLaughlin [3,4], on irreducible linear groups which contain transvection subgroups and also to work of Dempwolff's [1,2]. In [1,2] Dempwolff shows that an irreducible subgroup $Y$ of SL $(V)$ which is generated by 2 -transvections either contains transvections, has a normal abelian subgroup of odd order which has 1-dimensional homogeneous components on $V$ which $Y$ permutes transitively, contains a normal complex of Stellmacher elements, or a normal complex of roots involutions.

Theorem 1.1 generalizes a result due to Timmesfeld for the case $q=2$, which plays an important part in the proof of his Theorem 4.5 [9]. This more general theorem plays an equally vital role in the classification of symplectic amalgams [5]. The proof given here is modelled on Timmesfeld's approach. We remark that Theorem 1.1 is also the principal content of [7, Satz 3.10]. However, the proof there calls upon the (lengthy) classification of groups generated by root involutions and then deals with the resulting configurations case by case. Our proof is direct and elementary-the only substantial results we use being the classification of groups generated by transvections due to McLaughlin [3, 4].

Our notation follows that of [8]. The following elementary result will be used in the proof of Theorem 1.1.

LEMMA 1.2. Let $V$ be a vector space of dimension $2 n, n \geq 1$ which is equipped with a non-degenerate alternating bilinear form $f$. Put $G=\operatorname{Sp}(V)$. If $a$ and $b$ are non-zero vectors of $V$ with $f(a, b) \neq 0$, then

$$
G=\left\langle O_{2}\left(\operatorname{Stab}_{G}\langle a\rangle\right), O_{2}\left(\operatorname{Stab}_{G}\langle b\rangle\right)\right\rangle .
$$

PROOF. See [6]. 


\section{Orbits on points and vectors and a quadratic form}

From now on we assume the situation depicted in Theorem 1.1. If $L$ contains a transvection subgroup, then we may avail ourselves of McLaughlin's results $([3,4])$ to conclude, since $L \leq \operatorname{Sp}(V)$, that $L$ must be isomorphic to one of $\operatorname{Sp}(V), \mathrm{O}^{ \pm}(V), \operatorname{Sym}(2 n+1)$, or $\operatorname{Sym}(2 n+2)$. Of these groups only $\operatorname{Sp}(V)$ and $\mathrm{O}^{ \pm}(V)$ can contain an elementary abelian 2-group as large as $X$ and so Theorem 1.1 holds in this case. Henceforth, therefore, we shall suppose that $L$ contains no transvection subgroups.

LEMMA 2.1. (i) $\left[a^{\perp}, X\right]=\langle a\rangle$;

(ii) $[V, X]=a^{\perp}$;

(iii) $C_{V}(X)=\langle a\rangle$; and

(iv) Let $Z$ be a subgroup of index $q$ in $X$ and let $U=\langle a, c\rangle$ be a 2-dimensional subspace of $a^{\perp}$. If $Z$ centralizes $U$, then $C_{V}(Z)=U$.

PRoOF. Since $O_{2}(H) \geq X,\langle a\rangle=\left[a^{\perp}, O_{2}(H)\right] \geq\left[a^{\perp}, X\right]$ and part (i) follows as $X$ contains no transvections. Now suppose that $[V, X]<a^{\perp}$. Then $|[V, X]| \leq q^{2 n-2}$. So, letting $v \in V \backslash a^{\perp}, \mathscr{V}=\{[v, x] \mid x \in X\}$ contains at most $q^{2 n-2}$ vectors. If $|\mathscr{V}|=q^{2 n-2}$, then $\mathscr{V}=[V, X]$ and hence, by part (i), $[v, x]=a$, for some $x \in X$. But then $[V, x] \leq\left\langle\left[a^{\perp}, x\right],[v, x]\right\rangle \leq\langle a\rangle$, which implies that $x$ is a transvection on $V$, a contradiction. Thus $|\mathscr{V}|<q^{2 n-2}$. Since $|X|=q^{2 n-2}$, there must exist distinct $x_{1}, x_{2} \in X$ with $\left[v, x_{1}\right]=\left[v, x_{2}\right]$, whence $\left[v, x_{1} x_{2}\right]=0$. This then implies that $x_{1} x_{2}$ is a transvection on $V$. Hence we infer that $[V, X]=a^{\perp}$, and we have (ii). Using part (ii) gives $C_{V}(X)=[V, X]^{\perp}=\left(a^{\perp}\right)^{\perp}=\langle a\rangle$, so proving (iii).

For part (iv), since $Z$ centralizes $a$ and $c$, we have $[V, Z] \leq a^{\perp} \cap c^{\perp}=U^{\perp}$ with $\left|U^{\perp}\right|=q^{2 n-2}$. Now, as $|Z|=q^{2 n-3}$, we may argue as in part (ii) to obtain $[V, Z]=U^{\perp}$. Hence $C_{V}(Z)=[V, Z]^{\perp}=U$.

By hypothesis $L$ acts irreducibly upon $V$ and so $\left\langle a^{L}\right\rangle=V$. Thus we can find an image $\langle b\rangle$ of $\langle a\rangle$ (under $L$ ) with $b \in V \backslash a^{\perp}$. Select the vector representative $b$ of $\langle b\rangle$ so as $f(a, b)=1$. Then, for $\lambda \in k$ we put $\mathscr{O}_{\lambda}=\left\{\left\langle b^{x}+\lambda a\right\rangle \mid x \in X\right\}$, and note that each $\mathscr{O}_{\lambda}$ is an $X$-orbit which consists of points (1-spaces of $V$ ) in $V \backslash a^{\perp}$.

LEMMA 2.2. $X$ has $q$ regular orbits on the points in $V \backslash a^{\perp}$. These $X$-orbits are $\mathscr{O}_{\lambda}, \lambda \in k$.

PRoof. Suppose $\langle v\rangle$ is a point of $V \backslash a^{\perp}$ which is fixed by $x \in X^{\#}$. Then, by Lemma 2.1 (ii), $[\langle v\rangle, x] \leq[V, X] \cap\langle v\rangle=a^{\perp} \cap\langle v\rangle=0$. Hence

$$
[V, x]=\left[a^{\perp}+\langle v\rangle, x\right]=\left[a^{\perp}, x\right] \leq\langle a\rangle,
$$


a contradiction as $X$ contains no transvections. Therefore the $X$-orbits of points of $V$ not contained in $a^{\perp}$ are regular. So, by counting, we see that $X$ has exactly $q$ orbits on the points in $V \backslash a^{\perp}$. To complete the proof of the lemma we must show that for $\lambda, \mu \in k, \mathscr{O}_{\lambda}=\mathscr{O}_{\mu}$ implies that $\lambda=\mu$. Let $\lambda, \mu \in k$ be such that $\mathscr{O}_{\lambda}=\mathscr{O}_{\mu}$. Then $b+\lambda a=b^{x}+\mu a$ for some $x \in X$. So $[b, x]=b+b^{x}=(\mu+\lambda) a \in\langle a\rangle$ and consequently $[V, x] \leq\langle a\rangle$. Since no element of $X^{*}$ is a transvection on $V$, we must have $x=1$ and then $\lambda a=\mu a$. Hence $\lambda=\mu$.

For each $x \in X^{*}$ we define a 2-dimensional subspace $T_{x}$ of $a^{\perp}$ by

$$
T_{x}=[V, x]=\langle a,[b, x]\rangle=\langle a\rangle+\langle[b, x]\rangle .
$$

LEMMA 2.3. For $x \in X^{\prime \prime}$ we have $T_{x} \cap b^{\perp}=\left\langle[b, x]+f\left(b, b^{x}\right) a\right\rangle$.

PROOF. Since $\operatorname{dim} b^{\perp}=2 n-1$ and $\langle a\rangle \succeq b^{\perp}, \operatorname{dim}\left(T_{x} \cap b^{\perp}\right)=1$. Now

$$
\begin{aligned}
f\left(b,[b, x]+f\left(b, b^{x}\right) a\right) & =f\left(b, b+b^{x}\right)+f\left(b, b^{x}\right) f(b, a) \\
& =f(b, b)+f\left(b, b^{x}\right)+f\left(b, b^{x}\right)=0,
\end{aligned}
$$

and so $[b, x]+f\left(b, b^{x}\right) a \in T_{x} \cap b^{\perp}$. Thus, as $[b, x]+f\left(b, b^{x}\right) a \neq 0$, Lemma 2.3 holds.

LEMMA 2.4. Suppose $T$ is a 2-dimensional subspace of $a^{\perp}$ which contains $\langle a\rangle$. Then

(i) there exists $x \in X^{\prime \prime}$ such that $T_{x}=T$; and

(ii) $X_{T}:=\left\{x \in X^{*} \mid T_{x}=T\right\} \cup\{1\}$ is a subgroup of $X$ of order $q$.

PROOF. Let $T$ be a 2-dimensional subspace of $a^{\perp}$ containing $\langle a\rangle$, and suppose that $\left\{x \in X^{\#} \mid T_{x}=T\right\}$ has at least $q$ distinct elements. Then, since $\operatorname{dim}\left(T \cap b^{\perp}\right)=1$, Lemma 2.3 implies there exists $x_{1}, x_{2} \in X^{\#}$ with $x_{1} \neq x_{2}$ such that

$$
\left[b, x_{1}\right]+f\left(b, b^{x_{1}}\right) a=\left[b, x_{2}\right]+f\left(b, b^{x_{2}}\right) a .
$$

So $b+b^{x_{1}}+b+b^{x_{2}}=\left(f\left(b, b^{x_{1}}\right)+f\left(b, b^{x_{2}}\right)\right) a$, whence

$$
b+b^{x_{1} x_{2}^{-1}}=\left(f\left(b, b^{x_{1}}\right)+f\left(b, b^{x_{2}}\right)\right) a^{x_{2}^{-1}}=\left(f\left(b, b^{x_{1}}\right)+f\left(b, b^{x_{2}}\right)\right) a \in\langle a\rangle .
$$

This forces $x_{1} x_{2}^{-1}$ to be a transvection of $V$ and so we conclude that

$$
\left|\left\{x \in X^{*} \mid T_{x}=T\right\}\right| \leq q-1 .
$$

So there must be at least $(|X|-1) /(q-1)=\left(q^{2 n-2}-1\right) /(q-1)$ 2-dimensional subspaces of $V$ of the form $T_{x}$, for some $x \in X^{\#}$. However, this is also the number of 2-dimensional subspaces of $a^{\perp}$ which contain $\langle a\rangle$ and so (i) holds. Moreover, $\left|\left\{x \in X^{\#} \mid T_{x}=T\right\}\right|=q-1$. Since, for $x_{1}, x_{2} \in X_{T},\left[b, x_{1} x_{2}\right]=\left[b, x_{1}\right]^{x_{2}}+\left[b, x_{2}\right] \in$ $T$ we infer that $X_{T}$ is a subgroup of $X$ of order $q$. 
LEMMA 2.5. If $T$ is a 2-dimensional subspace of $a^{\perp}$ containing $\langle a\rangle$, then

(i) $\left|X / C_{X}(T)\right|=q$; and

(ii) $X$ has two orbits on the points of $T$, with one of length $q$ and one consisting of $\langle a\rangle$.

PROOF. By definition $T=T_{x}$ for all $x \in X_{T}^{\#}$. Thus $C_{V}(x)=[V, x]^{\perp}=T_{x}^{\perp}=T^{\perp}$. Hence $C_{V}\left(X_{T}\right)=T^{\perp}$. Select a 2-dimensional subspace $U$ of $a^{\perp}$ containing $\langle a\rangle$ so as $U+T^{\perp}=a^{\perp}$. Since $T^{\perp} \neq a^{\perp}, U^{\perp} \cap T=\langle a\rangle$. Because $C_{V}\left(X_{U}\right)=U^{\perp}$ we then deduce that $C_{T}\left(X_{U}\right)=\langle a\rangle$ and therefore $X_{U}$ has orbits of size 1 and $q$ on the points of $T$. Since $X / C_{X}(T)$ embeds into $\mathrm{GL}_{2}(q)$, this gives the lemma.

LEMMA 2.6. Suppose that $\langle c\rangle \leq a^{\perp}$ is in the same L-orbit as $\langle a\rangle$ and $\langle c\rangle \neq\langle a\rangle$. Then

(i) the non-zero vectors of $\langle a, c\rangle$ are all in the same $L$-orbit; and

(ii) $N_{L}(\langle a\rangle) / C_{L}(\langle a\rangle)$ has order $q-1$.

Proof. Put $T=\langle a, c\rangle$. By assumption $\langle c\rangle=\langle a\rangle^{g}$ for some $g \in L$. Applying Lemma 2.5 (i) to the pair $T,\langle c\rangle$ gives $\left|X^{g} / C_{X^{8}}(T)\right|=q$. Since $X$ and $X^{g}$ act differently on $T$, we deduce that $\left\langle X, X^{g}\right\rangle / C_{\langle X, X s\rangle}(T) \cong \mathrm{SL}_{2}(q)$, and this gives the result.

An immediate consequence of Lemma 2.6 is

LEMMA 2.7. If $L$ is transitive on the points of $V$, then $L$ is transitive on $V^{\#}$.

LEMMA 2.8. Suppose that $L$ is not transitive on the points of $V$. Then there exists a 2-dimensional subspace $T$ of $a^{\perp}$ containing $\langle a\rangle$ such that $\langle a\rangle^{L} \cap T=\{\langle a\rangle\}$. Moreover, for $U=\left\langle b, T \cap b^{\perp}\right\rangle, N_{X}(U)=1$.

PROOF. Suppose no such 2-dimensional subspace of $a^{\perp}$ exists. Then all points in $a^{\perp}$ are in $\langle a\rangle^{L}$ by Lemma 2.6. Let $\langle d\rangle$ be a point of $V$ not in $a^{\perp}$. Since $\operatorname{dim} V \geq 4$, there exists $0 \neq c \in a^{\perp} \cap d^{\perp}$ whence $\langle c\rangle \in\langle a\rangle^{L}$. And then $\langle d\rangle \in\langle a\rangle^{L}$. So $L$ is transitive on the points of $V$, a contradiction.

We now let $T$ be such a 2-dimensional subspace and prove that, for $U=\left\langle b, T \cap b^{\perp}\right\rangle$, $N_{X}(U)=1$. Let $1 \neq x \in X$. We claim that $[b, x] \notin T \cap b^{\perp}$. For if $[b, x] \in T \cap b^{\perp}$, then $\langle b\rangle \leq\langle[b, x], b\rangle=\left\langle b^{x}, b\right\rangle \leq b^{\perp}$. Since $\left\langle b^{x}, b\right\rangle$ is a 2-dimensional subspace by Lemma 2.1 (ii), we may apply Lemma 2.6 (with $b$ in place of $a$ ) to conclude that $\langle[b, x]\rangle \in\langle b\rangle^{L}$. Hence, as $\langle a\rangle$ and $\langle b\rangle$ are in the same $L$-orbit, $\langle[b, x]\rangle \in\langle a\rangle^{L} \cap T=$ $\{\langle a\rangle\}$, a contradiction which establishes the claim. Since $b \notin T, U$ is a 2-dimensional subspace and $U \cap a^{\perp}=T \cap b^{\perp}$. If there exists $1 \neq x \in N_{X}(U)$, then, since $\langle b\rangle^{X}$ is a regular $X$-orbit, $\left\langle b, b^{x}\right\rangle=U$ and consequently $[b, x] \in U \cap a^{\perp}=T \cap b^{\perp}$, contrary to $[b, x] \notin T \cap b^{\perp}$. Therefore, $N_{X}(U)=1$. 
LEMMA 2.9. Either $L$ is transitive on the points of $V$ or $L$ has two orbits on the points of $V$. In the latter case we have

$$
\langle a\rangle^{L} \backslash a^{\perp}=\langle b\rangle^{X}=\mathscr{O}_{0} \text { and }\langle a+b\rangle^{L} \backslash a^{\perp}=\bigcup_{\lambda \in k^{\prime \prime}} \mathscr{O}_{\lambda}
$$

PROOF. Suppose that $L$ is not transitive on the points of $V$. Then, by Lemma 2.8, there exists a 2-dimensional subspace $T$ with $\langle a\rangle \leq T \leq a^{\perp}$ and $\langle a\rangle^{L} \cap T=\{\langle a\rangle\}$. Setting $U=\left\langle b, T \cap b^{\perp}\right\rangle$ we also have $N_{X}(U)=1$ by Lemma 2.8. Since the elements of $X^{*}$ all have order 2, it follows that no two points of $U \backslash a^{\perp}$ are in the same $X$-orbit. Hence the $q$ points of $U \backslash a^{\perp}$ may be chosen as representative of the $X$-orbits on $V \backslash a^{\perp}$. Let $g \in L$ be such that $\langle a\rangle^{g}=\langle b\rangle$, and set $Y=X^{g}$. Then, by Lemma 2.5 (ii), $Y$ has orbits of length 1 and $q$ on the points of $U$ and so, as $\langle b\rangle^{\gamma}=\{\langle b\rangle\}, \bigcup_{\lambda \in k^{\prime \prime}} \mathscr{O}_{\lambda}$ is contained in an $L$-orbit.

For $\langle c\rangle \leq a^{\perp}$, if $\langle c\rangle \notin\langle a\rangle^{L}$, then we may use the above argument with $\langle a, c\rangle$ in place of $T$ to show that $\langle c\rangle$ is in the same $L$-orbit as $\bigcup_{\lambda \in k^{*}} O_{\lambda}$. Thus $L$ has two orbits on the points of $V$ with $\langle a\rangle^{L} \backslash a^{\perp}=\mathscr{O}_{0}$ and $\langle a+b\rangle^{L} \backslash a^{\perp}=\bigcup_{\lambda \in k^{\prime \prime}} \mathscr{O}_{\lambda}$, and the lemma is proven.

So far we have been dealing with points of $V$. The next lemma tells us about the orbits of $L$ on the vectors of $V$. As will shortly be seen this plays an important role in the remainder of the proof of Theorem 1.1.

LEMMA 2.10. Suppose that $L$ is not transitive on the points of $V$. Then

(i) the vectors of $\langle a+b\rangle$ are in distinct $L$-orbits; and

(ii) the vectors of $\langle a\rangle^{\prime \prime}$ are in a single $L$-orbit.

PROOF. Clearly, if $q=2$ there is nothing to prove, so we may assume $q>2$. Again we choose a 2-dimensional subspace $T$ with $\langle a\rangle \leq T \leq a^{\perp}$ and $\langle a\rangle^{L} \cap T=\{\langle a\rangle\}$. Let $x \in X^{*}$ be such that $T=T_{x}$. Then, by Lemma 2.3, $c=[b, x]+f\left(b, b^{x}\right) a \in T \cap b^{\perp}$. Put $U=\langle b, c\rangle$. Then $T$ and $U$ are both subspaces of $c^{\perp}$ which contain $\langle c\rangle$. Let $g \in L$ be such that $\langle a\rangle^{g}=\langle b\rangle$, and set $Y=X^{g}$. Put $K=\left\langle C_{X}(c), C_{Y}(c)\right\rangle$ and $W=c^{\perp} /\langle c\rangle$. By Lemma 2.5 (i), $\left|C_{X}(c)\right|=\left|C_{Y}(c)\right|=q^{2 n-3}$ and clearly $K$ acts upon $W$. From Lemma $2.8 N_{X}(U)=1$ and so, as $\langle c\rangle \leq U \leq c^{\perp}, C_{C_{X}(c)}(W)=1$. Likewise $C_{C_{Y}(c)}(W)=1$. Put $\bar{K}=K / C_{K}(W)$. By orders $\overline{C_{X}(c)}$ and $\overline{C_{Y}(c)}$ are, respectively, the largest normal 2-subgroups of the stabilizer, in $\bar{K}$, of $a+\langle c\rangle$ and $b+\langle c\rangle$. Since $f(a+\langle c\rangle, b+\langle c\rangle)=1$, using Lemma 1.2 we deduce that $\bar{K} \cong \operatorname{Sp}(W)$. Because $L$ is assumed to contain no transvection subgroups, the structure of $C_{G}(c)$ and $q>2$ imply that $O_{2}\left(C_{L}(c)\right)=1$. So $C_{K}(W)=1$ and hence $K \cong \mathrm{Sp}(W)$. Since $\operatorname{Sp}(W)$ acts transitively upon the non-zero vectors of $W$ and $\langle a\rangle^{L} \cap T=\{\langle a\rangle\}$, it follows that the vectors of $\langle a\rangle^{\#}$ are in the same $L$-orbit, so (ii) holds. If (i) were 
false, then there would exist an element in $L$ acting non-trivially upon $\langle c\rangle$. Hence, as $K \cong \mathrm{Sp}(W)$ and $O_{2}\left(C_{L}(c)\right)=1, \operatorname{Stab}_{L}(\langle c\rangle)=Z \times K$ with $Z \neq 1$ and $Z$ centralizing $W$. Noting that $|Z|$ divides $(q-1)$ and $Z$ must act non-trivially on $V / c^{\perp}$, we see that $V=[V, Z] \times C_{V}(Z)$ with $\operatorname{dim}[V, Z]=2$ and $K$ inducing the full symplectic group on $C_{V}(Z)$. Since $K$ is a simple group (recall $q>2$ ) and $K$ centralizes $c \in[V, Z]$, it follows that $K$ centralizes $[V, Z]$. But then $K$, and hence $L$, contains a transvection subgroup. Thus we conclude that (i) also holds.

LEMMA 2.11. Suppose that $L$ has two orbits on the points of $V$. Then for $\lambda, \mu \in$ $k, \mu \neq 0$, the vectors $\lambda a+\mu b$ and $\lambda \mu a+b$ are in the same $L$-orbit.

PROOF. Since all the non-zero vectors of $\langle a\rangle$ are in the same $L$-orbit by Lemma 2.10 (ii), $a^{g}=\mu a$ for some $g \in L$. So $g$ stabilizes $\langle a\rangle$ and hence leaves $a^{\perp}$ invariant. Consequently $g$ leaves invariant $\langle a\rangle^{L} \backslash a^{\perp}=\langle b\rangle^{X}$, using Lemma 2.9. So for a suitable $x \in X, g x$ stabilizes $\langle b\rangle$. Set $g_{1}=g x$, and notice that $a^{g_{1}}=a^{g x}=\mu a^{x}=\mu a$. Now $1=f(a, b)=f\left(a^{g_{1}}, b^{g_{1}}\right)=f\left(\mu a, b^{g_{1}}\right)=f\left(a, \mu b^{g_{1}}\right)$. Since $\mu b^{g_{1}} \in\langle b\rangle$, we must have $\mu b^{g_{1}}=b$ by the original choice of $b$. So $b^{g_{1}}=\mu^{-1} b$ and then

$$
(\lambda a+\mu b)^{g_{1}}=\lambda a^{g_{1}}+\mu b^{g_{1}}=\lambda \mu a+\mu \mu^{-1} b=\lambda \mu a+b,
$$

which gives the result.

For the moment we suppose that $L$ has two orbits on the points of $V$. For $v \in V$ define $Q: V \rightarrow k$ by

$$
Q(v)= \begin{cases}0 & \text { if } v \in a^{L} \cup\{0\} \\ \lambda^{2} & \text { if } v \in(\lambda a+\lambda b)^{L} .\end{cases}
$$

In view of Lemma 2.10, $Q$ is well defined. Observe that for $v=\mu a+b, \mu \neq 0, v$ is in $(\sqrt{\mu} a+\sqrt{\mu} b)^{L}$ by Lemma 2.11 and therefore $Q(v)=\mu$. Also for all $\mu \in k, v \in V$, we have $Q(\mu u)=\mu^{2} Q(v)$. It is immediate from its definition that $Q$ is $L$-invariant.

LEMMA 2.12. Assume that $v \in a^{L}$. Then for $w \in V$ and $\alpha, \beta \in k$

$$
Q(\alpha v+\beta w)=\alpha^{2} Q(v)+\beta^{2} Q(w)+\alpha \beta f(v, w) .
$$

PROOF. Clearly, if $\beta=0$ then we are done-so we assume $\beta \neq 0$. Without loss of generality we may suppose that $v=a$. Suppose first that $w \in a^{\perp}$. In this case we need to show that $Q(\alpha a+\beta w)=\beta^{2} Q(w)=Q(\beta w)$. This means we need to show that $\alpha a+\beta w$ is in the same $L$-orbit as $\beta w$. If $w \in\langle a\rangle$, then $Q(\alpha a+\beta w)=0=Q(\beta w)$. Therefore, we may also assume $w \notin\langle a\rangle$ and so $\langle w, a\rangle$ is a 2-dimensional subspace of $a^{\perp}$. By Lemma 2.5 (i) $X / C_{X}(\langle w, a\rangle)$ has order $q$ and hence there exists $x \in X$ such 
that $[\beta w, x]=\alpha a$. Thus $\beta w+\beta w^{x}=\alpha a$, which is to say that $\beta w=(\alpha a+\beta w)^{x}$ and we are done.

Next we suppose that $w \notin a^{\perp}$. Then we have two cases, the first being when $w \in a^{L}$. Here we have $w=\tau b^{x}$ for some $\tau \in k(\tau \neq 0), x \in X$ by Lemma 2.9. Hence, as $X$ fixes $a, \alpha a+\beta w=\alpha a+\beta \tau b^{x}=(\alpha a+\beta \tau b)^{x}$. Therefore, by Lemma 2.11, $\alpha a+\beta w$ is in the same $L$-orbit as $\alpha \beta \tau a+b$, whence $Q(\alpha a+\beta w)=\alpha \beta \tau$. Now $f(a, w)=f\left(a, \tau b^{x}\right)=f(a, \tau b)=\tau f(a, b)=\tau$. Therefore,

$$
\alpha^{2} Q(a)+\beta^{2} Q(w)+\alpha \beta f(a, w)=\alpha^{2} 0+\beta^{2} 0+\alpha \beta \tau=\alpha \beta \tau=Q(\alpha a+\beta w),
$$

as required. Turning to the second case, $w \notin a^{\perp}$, again using Lemma 2.9 we have $w=\tau b^{x}+\tau \lambda a$, for some $\lambda, \tau \in k(\tau \neq 0 \neq \lambda), x \in X$. So, by Lemma 2.11, $\alpha a+\beta w$ and $\beta \tau(\alpha+\beta \tau \lambda) a+b$ are in the same $L$-orbit and hence $Q(\alpha a+\beta w)=\beta \tau(\alpha+\beta \tau \lambda)$. Because $w$ and $\tau b+\tau \lambda a$ are in the same $L$-orbit, we obtain $Q(w)=\tau^{2} \lambda$. Since $Q(a)=0$ and $f(a, w)=\tau$,

$$
\alpha^{2} Q(a)+\beta^{2} Q(w)+\alpha \beta f(a, w)=\beta^{2} \tau^{2} \lambda+\alpha \beta \tau=Q(\alpha a+\beta w) .
$$

This verifies the equation in the final case and so the lemma holds.

LEMMA 2.13. $Q$ is a quadratic form on $V$.

Proof. Let $v, w \in V$ and $\alpha, \beta \in k$. So we must show that $Q(\alpha v+\beta w)=$ $\alpha^{2} Q(v)+\beta^{2} Q(w)+\alpha \beta f(v, w)$. In view of Lemma 2.12 we only need examine the case when $v$ and $w$ are not in the same $L$-orbit as $a$. Also, we may assume that $w \notin a^{\perp}$. Then, by Lemma 2.2, $w \in \mathscr{O}_{\lambda}$ for some $\lambda \in k^{*}$. Hence $w=b^{x}+\lambda a$ for some $x \in X$. Therefore, $w+\lambda a=b^{x} \in a^{L}$ and so, using Lemma 2.12,

$$
\begin{aligned}
Q(\alpha v+\beta w)= & Q(\alpha v+\beta \lambda a+\beta w+\beta \lambda a) \\
= & Q(\alpha v+\beta \lambda a)+Q(\beta w+\beta \lambda a)+f(\alpha v+\beta \lambda a, \beta w+\beta \lambda a) \\
= & \alpha^{2} Q(v)+\beta^{2} \lambda^{2} Q(a)+\alpha \beta \lambda f(v, a)+\beta^{2} Q(w) \\
& +\beta^{2} \lambda^{2} Q(a)+\beta^{2} \lambda f(w, a)+\alpha \beta f(v, w) \\
& +\alpha \beta \lambda f(v, a)+\beta^{2} \lambda f(a, w)+\beta^{2} \lambda^{2} f(a, a) \\
= & \alpha^{2} Q(v)+\beta^{2} Q(w)+\alpha \beta f(v, w) .
\end{aligned}
$$

This proves the lemma.

LEMMA 2.14. If $L$ has two orbits on the points of $V$, then $L$ acts naturally on $V$ as one of $\Omega^{ \pm}(V)$ and $\mathrm{O}^{ \pm}(V)$.

ProOF. From Lemma 2.13 $L$ preserves the quadratic form $Q$ and so $L$ is a subgroup of $\mathrm{O}(V, Q)\left(\cong \mathrm{O}^{ \pm}(V)\right)$. Now $X=O_{2}\left(\operatorname{Stab}_{O(V, Q)}\langle a\rangle\right)$ and the orbits of $L$ and $\mathrm{O}(V, Q)$ upon the points of $V$ are the same. Hence $L \geq\left\langle X^{O(V, Q)}\right\rangle \geq \Omega^{ \pm}(V)$. Thus Lemma 2.14 holds. 


\section{Proof of Theorem 1.1}

In the light of Lemma 2.14 from now on we may assume that $L$ is transitive on the points of $V$, whence, by Lemma 2.6, $L$ is transitive on $V^{\#}$. We recall there is a one-toone correspondence between vectors $c$ of $V$ and symplectic transvections $\tau_{c}$ of $\operatorname{Sp}(V)$ where $\tau_{c}$ is defined by $\tau_{c}(v)=v+f(v, c) c(v \in V)$. Hence $L$ acts transitively (by conjugation) upon the symplectic transvections. Therefore if $L$ contains a transvection it must contain them all and, in particular, will then contain a transvection subgroup. So we conclude that $L$ contains no transvections. For $c$ a non-zero vector of $V$ we define $X_{c}$ to be $X^{g}$ where $g \in L$ is such that $a^{g}=c$. If $a^{g_{1}}=c$ for $g_{1} \in L$, then $X^{g} X^{g_{1}} \leq O_{2}\left(\operatorname{Stab}_{G}\langle c\rangle\right)$ and if $X^{g} \neq X^{g_{1}}$, then $X^{g} X^{g_{1}}$ intersects the transvection subgroup of $O_{2}\left(\operatorname{Stab}_{G}\langle c\rangle\right)$ non-trivially. So $X_{c}$ is well defined; note that $X_{a}=X$. We now complete the proof of Theorem 1.1, arguing by induction on $n$ and starting with $n=2$. When $q=2$ we have $G \cong \operatorname{Sym}(6)$ and it is fairly straightforward to calculate in Sym(6) to deduce that $L=\operatorname{Alt}(6)$ or $\Gamma \mathrm{L}(2,4)$. Thus we may assume $q>2$. We note that $\left|X_{a}\right|=|X|=q^{2}$. Let $T$ be a 2-dimensional space of $a^{\perp}$ containing $\langle a\rangle$, and let $t \in T \backslash\langle a\rangle$. From Lemmas 2.4 (ii) and 2.5 (i) we have that $X_{T}=C_{X_{a}}(T)$ has order $q$. Put $Y_{a t}=\left\langle X_{a}, X_{t}\right\rangle$. Then $Y_{a t} \leq \operatorname{Stab}_{G} T \sim q^{1+2}\left(\mathrm{SL}_{2}(q) \times(q-1)\right)$. Recall that $\mathrm{O}_{2}\left(\operatorname{Stab}_{G}(T)\right)$ is a indecomposable $\mathrm{GF}(q) \mathrm{Stab}_{G}(T)$-module. Hence, since $Y_{a t} \leq O^{2^{\prime}}\left(\operatorname{Stab}_{G}(T)\right)$ and $L$ contains no transvections, $Y_{a t} \cong q \times \mathrm{SL}_{2}(q)$ with $Y_{a t} \cap O_{2}\left(\operatorname{Stab}_{G}(T)\right)=X_{T}$ having order $q$. Evidently $X_{a} \in \operatorname{Syl}_{2} Y_{a t}$. Furthermore $N_{Y_{a t}}\left(X_{a}\right)$ has order $q^{2}(q-1)$, stabilizes $\langle a\rangle$ and induces $\left\{\left(\begin{array}{ll}\lambda & 0 \\ 0 & 1\end{array}\right) \mid \lambda \in \mathrm{GF}(q)\right\}$ on $X_{a}$. For 2-dimensional subspaces $T_{1}, T_{2}$ of $a^{\perp}$ containing $\langle a\rangle$ with $T_{1} \neq T_{2}$ we must have $X_{T_{1}} \neq X_{T_{2}}$ for otherwise $X_{T_{1}}=X_{T_{2}}$ centralizes $T_{1}+T_{2}=a^{\perp}$. Thus

$$
\left.N=\left\langle N_{Y_{a r}}\left(X_{a}\right)\right| T=\langle t, a\rangle \text { a subspace of } a^{\perp}\right\rangle\left(\leq \operatorname{Stab}_{G}\langle a\rangle\right)
$$

induces a 2-transitive action upon the points of $X_{a}$, and consequently $N / C_{N}\left(X_{a}\right)$ $\cong \mathrm{SL}_{2}(q) \times(q-1)$. So, as $L$ has no transvections, $N \sim q^{2}\left(\mathrm{SL}_{2}(q) \times(q-1)\right)$. But this contradicts the structure of $\operatorname{Stab}_{G}\langle a\rangle$ and therefore we have verified the theorem for $n=2$.

From now on we assume that $n \geq 3$. Again we consider a 2-dimensional subspace $T$ of $a^{\perp}$ which contains $\langle a\rangle$, and let $t \in T \backslash\langle a\rangle$. Put $X_{T, t}=\left\langle x \in X_{t} \mid[V, t]=T\right\rangle$, and let $K_{T, t}$ be a complement to $X_{T, t}$ in $C_{X_{t}}(T)$. By Lemmas 2.4 and 2.5 (i) $\left|K_{T, t}\right|=q^{2 n-4}$. We next show that

3.1. (i) $\left|K_{T, t} O_{2}(H) / O_{2}(H)\right|=q^{2 n-4}$;

(ii) $K_{T, t} O_{2}(H)$ centralizes $T^{\perp} / T$; and

(iii) no element of $K_{T, t}$ induces a transvection on $a^{\perp} /\langle a\rangle$.

Suppose that $1 \neq x \in K_{T, t} \cap O_{2}(H)$. Then $\left[a^{\perp}, x\right] \leq\langle a\rangle$ and $\left[t^{\perp}, x\right] \leq\langle t\rangle$. Since $x$ is not a transvection on $V$ this gives $[V, x]=T$ which implies $x \in K_{T, t} \cap X_{T, t}=1$, a 
contradiction. Therefore part (i) holds. Part (ii) follows from $\left[T^{\perp}, K_{T, t}\right] \leq\left[t^{\perp}, X_{t}\right] \leq$ $\langle t\rangle \leq T$. Finally suppose that $1 \neq x \in K_{T, t}$ operates as a transvection on $a^{\perp} /\langle a\rangle$, and let $W$ be the preimage of $C_{a^{\perp} /\langle a\rangle}(x)$. Then $[W, x] \leq\langle a\rangle$. Assume that $[W, x]=\langle a\rangle$ holds. Since $x$ is a 2-transvection on $V$ and $x \in X_{t}$, this gives $[V, x]=T$ and so $x \in K_{T, t} \cap X_{T, t}=1$, a contradiction. Therefore [W, $\left.x\right]=0$ and so $W=C_{V}(x) \leq t^{\perp}$. Hence $C_{V}(x)=W=t^{\perp} \cap a^{\perp}=T^{\perp}$. But then $[V, x]=T^{\perp \perp}=T$ which again gives the impossible $x \in K_{T, t} \cap X_{T, t}=1$. Thus (iii) holds. Put $\bar{a}=a^{\perp} /\langle a\rangle$ and

$$
\left.L_{0}=\left\langle C_{X_{t}}(T)\right| t \in T \backslash\langle a\rangle \text { and } T \text { a 2-dimensional subspace of } a^{\perp} \text { containing }\langle a\rangle\right\rangle \text {. }
$$

\section{2. $L_{0}$ acts irreducibly upon $\bar{a}$.}

Suppose that $W$ is an $L_{0}$-invariant subspace with $\langle a\rangle<W<a^{\perp}$. Let $c \in a^{\perp}$ be a vector not in $W$, and put $U=\langle a, c\rangle$. Then, by Lemma 2.1 (i),

$$
\left[c^{\perp} \cap W, C_{X_{c}}(U)\right] \leq W \cap\langle c\rangle=0 .
$$

Therefore $\langle a\rangle \leq c^{\perp} \cap W \leq C_{V}\left(C_{X_{c}}(U)\right)$. Hence as $c \notin W, c^{\perp} \cap W=\langle a\rangle$ by Lemma 2.1 (iv) and so $\operatorname{dim} \bar{W}=1$. Thus every proper non-zero $L_{0}$-invariant subspace of $\bar{a}$ has dimension one. Since $\bar{W}^{\perp}$ is $L_{0}$-invariant, this forces $\bar{W}=\bar{W}^{\perp}$, whence $\operatorname{dim} V=4$. However we have $\operatorname{dim} V>4$, and so (3.2) holds.

Together (3.1) and (3.2) imply that $L_{0}$ acting on the 2(n-1)-dimensional symplectic space $\bar{a}$ satisfies the hypotheses of the theorem. Therefore, by induction, $L_{0} / C_{L_{0}}(\bar{a})$ is isomorphic to one of $\mathrm{O}^{ \pm}(\bar{a}), \Omega^{ \pm}(\bar{a}), \operatorname{Sp}(\bar{a})$, Alt $(6)$, or $\Gamma \mathrm{L}(2,4)$ with $q=n-$ $1=2$ in the latter two cases. The orthogonal cases are impossible as for every 1-dimensional subspace $\bar{T}$ of $\bar{a}$ there is a corresponding subgroup of order $q^{2 n-4}$ centralizing $T^{\perp} / T$ which is not the case in the orthogonal groups when we select a nonisotropic 1-dimensional subspace (stabilizer $\operatorname{Sp}_{2 n-4}(q)$ ). Thus $L_{0} / C_{L_{0}}(\bar{a}) \cong \operatorname{Sp}(\bar{a})$ or $L_{0} / C_{L_{0}}(\vec{a}) \cong \operatorname{Alt}(6)$ and $q=n-1=2$. Now $L$ containing no transvections forces $L_{0}$ to have index $q$ (or 4 in the $L_{0} / C_{L_{0}}(\bar{a}) \cong \operatorname{Alt}(6)$ case) in $H$ both of which are impossible. Therefore, we have that $L_{0} / C_{L_{0}}(\bar{a}) \cong \Gamma \mathrm{L}(2,4)$ and $L_{0} \sim 2^{4} \Gamma \mathrm{L}(2,4)$ with $O_{2}\left(L_{0}\right)$ a 'natural' $\mathrm{GF}(2) \Gamma \mathrm{L}(2,4)$ module. However, this means that $L_{0}$ is a subgroup of index 12 in $H \sim 2^{1+4} \mathrm{Sp}(4,2)$, and, since it has order coprime to 11 , has an orbit of length at most 10 on its twelve right cosets in $H$. By considering an orbit on which the $O_{2}\left(L_{0}\right)$ acts non-trivially we find that $L_{0}$ must have a subgroup of index 10 , which intersects $O_{2}\left(L_{0}\right)$ in a subgroup of order 8 and projects to $\operatorname{Sym}(4)$ in $L_{0} / O_{2}\left(L_{0}\right)$. This contradicts the module structure of $O_{2}\left(L_{0}\right)$ and thus $L_{0} / C_{L_{0}}(\bar{a}) ¥ \Gamma \mathrm{L}(2,4)$. This completes the proof of Theorem 1.1.

\section{References}

[1] U. Dempwolff, 'Some subgroups of SL $\left(n, 2^{m}\right)$, I', Results Math. 4 (1981), 1-21. 
[2] —, 'Some subgroups of SL $\left(n, 2^{m}\right)$, II', preprint 41, (Universität Kaiserslautern, 1982).

[3] J. McLaughlin, 'Some groups generated by transvections', Arch. Math. 18 (1967), 364-368.

[4] - 'Some subgroups of $\mathrm{SL}_{n}\left(F_{2}\right)$ ', Illinois J. Math. 13 (1969), 108-115.

[5] C. Parker and P. Rowley, Symplectic amalgams, Springer Monographs in Math., (Springer, New York, 2002).

[6] S. Smith, 'Spin modules in characteristic 2', J. Algebra 77 (1982), 392-401.

[7] G. Stroth, 'Endliche Gruppen, die eine Maximale 2-lokale Untergruppe besitzen, so daß $Z\left(F^{*}(M)\right)$ eine $T I$-Menge in $G$ ist', J. Algebra 64 (1980), 460-528.

[8] M. Suzuki, Group theory I, Grundlehren Math. Wiss. 247 (Springer, New York, 1982).

[9] F. G. Timmesfeld, 'Finite simple groups in which the generalized fitting subgroup of the centralizer of some involution is extraspecial', Ann. of Math. (2) 107 (1978), 297-369.

School of Mathematics and Statistics

University of Birmingham

Edgbaston

Birmingham B15 2TT

United Kingdom

e-mail: cwp@for.mat.bham.ac.uk
Department of Mathematics

UMIST

P.O. Box 88

Manchester M60 1QD

United Kingdom

e-mail: peter.rowley@umist.ac.uk 
